\title{
Secondary Normal-Pressure Hydrocephalus in Rheumatoid Meningitis
}

\author{
Micaela Owens ${ }^{a} \quad$ Na Tosha Gatson ${ }^{a}$ Gino Mongelluzzob ${ }^{b}$ Oded Goren ${ }^{c}$ \\ Eric Newman ${ }^{d}$ Mihai Cosmin Sandulescu ${ }^{a}$ \\ aDepartment of Neurology, Geisinger Medical Center, Danville, PA, USA; ${ }^{b}$ Department of \\ Radiology, Geisinger Medical Center, Danville, PA, USA; 'Department of Neurosurgery, \\ Geisinger Medical Center, Danville, PA, USA; dDepartment of Rheumatology, Geisinger \\ Medical Center, Danville, PA, USA
}

\section{Keywords}

Gait instability · Secondary normal pressure hydrocephalus $\cdot$ Rheumatoid meningitis

\begin{abstract}
Normal-pressure hydrocephalus (NPH) is a common cause of gait apraxia, cognitive impairment, and urinary incontinence in the elderly. It is usually a primary idiopathic disorder but can be secondary. We present a case of secondary NPH due to biopsy-confirmed rheumatoid meningitis initially refractory to intravenous (IV) immunotherapy. Our patient reported an excellent response right after shunting. Her gait remains normal one and a half years later. We searched PubMed for similar cases of rheumatoid meningitis with gait abnormality for additional clinicopathologic discussion. The patient's movement disorder initially improved with steroid taper. However, she developed progressive symptoms, later on, refractory to IV solumedrol and rituximab. She underwent ventriculoperitoneal shunting (VPS) and reported an outstanding outcome. This is the first reported biopsy-confirmed case of rheumatoid meningitis causing NPH to undergo shunting for immediate improvement. Previous cases of rheumatoid meningitis-associated Parkinsonism have improved with steroid induction. Although our patient's rheumatoid arthritis is now controlled, her case illustrates that NPH in autoinflammatory conditions may not recover with immune suppression alone. VPS is an option for a faster response in secondary NPH due to rheumatoid meningitis or other inflammatory disorders with progressive symptoms despite standard induction therapy.
\end{abstract}




\section{Introduction}

Normal-pressure hydrocephalus (NPH) is a common cause of gait apraxia, cognitive impairment, and urinary incontinence in the elderly. It is usually a primary idiopathic disorder but can be secondary to trauma, hemorrhage, neoplasm, and meningitis. We present a case of secondary NPH due to rheumatoid meningitis, with a discussion of the pathophysiology of secondary NPH and rheumatoid meningitis specifically. Our case proved initially refractory to IV immunotherapy but reported an excellent immediate response to shunting and sustained normal walking one year later.

\section{Case Report/Case Presentation}

A 77-year-old female with a past medical history of cutaneous squamous cell carcinoma and rheumatoid arthritis diagnosed 5 years previously presented to neurology for evaluation of gait abnormality. Current medications for rheumatoid arthritis included methotrexate, sulfasalazine, hydroxychloroquine, and chronic prednisone. She reported memory problems and gait abnormality since 2013, which progressed in 2017 to being wheelchair user. MRI, performed April 2018, revealed predominantly frontoparietal and symmetric sulcal T2/fluidattenuated inversion recovery signal with corresponding thick leptomeningeal enhancement. Ventriculomegaly was noted with an Evans' ratio of 0.36 and minimal periventricular fluidattenuated inversion recovery hyperintensity with a "capping" pattern (Fig. 1a, b). Outside lumbar puncture was unremarkable. Symptoms initially improved with steroid taper to nearnormal neurologic exam per history.
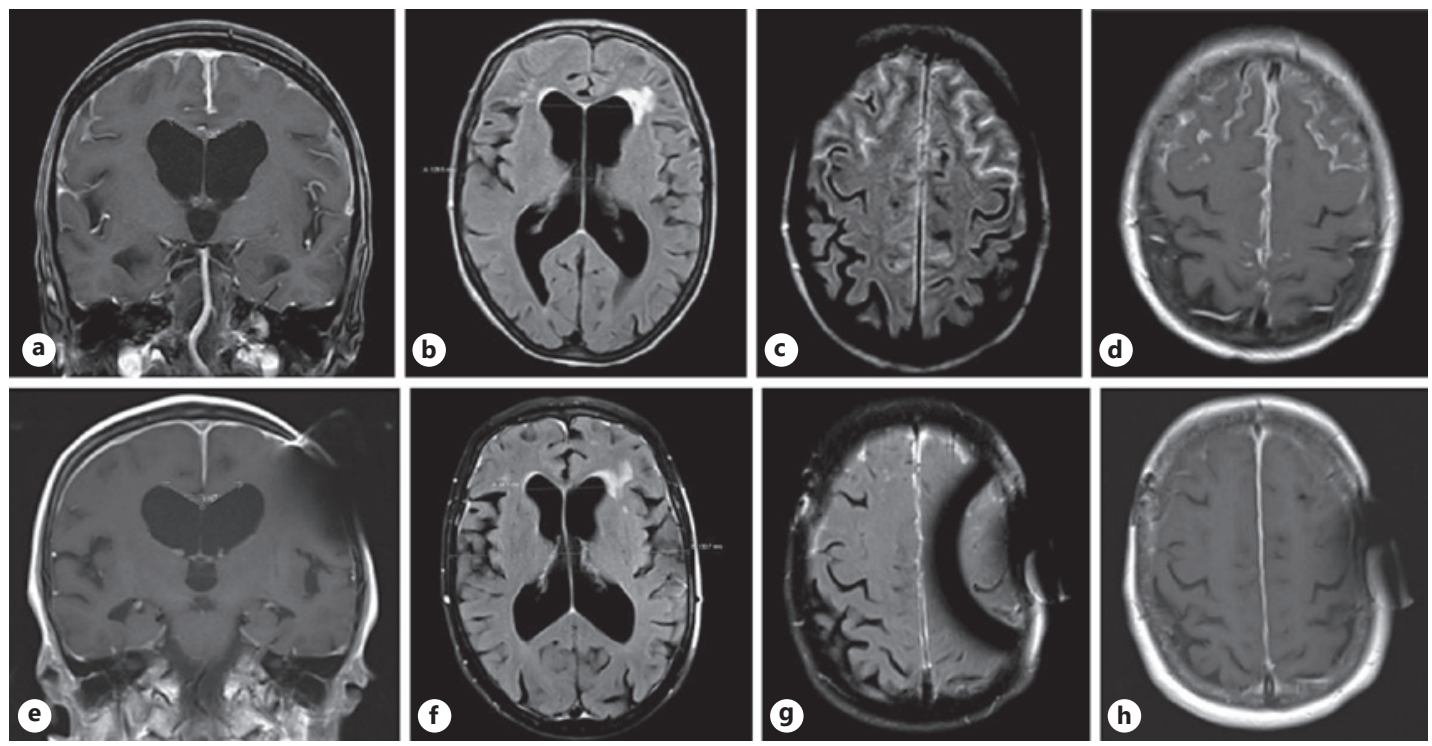

Fig. 1. Brain MRI sections before shunt placement (a-d) and 1 year after IV immunotherapy (e-h). a Coronal T1 post-contrast image showing leptomeningeal enhancement. b Axial T2-FLAIR image demonstrating increased ER of 47/129.5 = 0.36. $\mathbf{c}$ Axial T2-FLAIR and $\mathbf{d}$ post-contrast T1 images demonstrate increased sulcal hyperintensity and frontal-predominant leptomeningeal enhancement. $\mathbf{e}$ Coronal and $\mathbf{h}$ axial post-contrast T1 images indicate less leptomeningeal enhancement. f Axial T2-FLAIR illustrates a smaller ER 42/131=0.32 and $\mathbf{g}$ a decreased sulcal hyperintensity along with susceptibility artifact from shunt valve. IV, intravenous; FLAIR, fluid-attenuated inversion recovery; ER, Evans' ratio. 
Upon presentation to the movement disorders clinic in June 2018, she reported worsening gait with recurrent falling. She denied meningitis symptoms but did have active synovitis and a new diagnosis of chronic cystitis. Neurological examination revealed 27/30 on MOCA testing, vibratory sensation loss in both feet, mild bilateral upper extremity action tremor, and unsteady gait with a variably broad base, short stride, and impaired tandem. Neurological examination deteriorated in August 2018, with new bradykinesia, cogwheeling rigidity, decreased arm swing, positive pull test, Mini-Mental State Examination (MMSE) of 26/30, and Timed Up and Go Test (TUG) of $18 \mathrm{~s}$. She trialed carbidopa-levodopa without improvement. The patient's brain MRI remained stable (not shown). The extended serologic and infectious workup was unremarkable. Repeat lumbar puncture (August 2018) revealed opening pressure $16 \mathrm{~cm} \mathrm{H}_{2} \mathrm{O}$, normal cell counts, normal protein, and negative rheumatoid factor (RF). Repeat serum RF and cyclic citrullinated peptide autoantibodies were elevated to $29 \mathrm{IU} / \mathrm{mL}$ and $>250$ units, respectively, conferring high suspicion for rheumatoid meningitis despite bland cerebrospinal fluid (CSF). She underwent a meningeal biopsy before IV solumedrol, confirming granulomatous inflammation consistent with rheumatoid meningitis, as discussed below.

Solumedrol (September), acetazolamide, and rituximab (2 doses, October) made no difference in her neurological examination. Joint and bladder symptoms improved after rituximab therapy. After the first dose of rituximab, her neurological evaluation revealed an improved MMSE 30/30, but worse TUG, with an average of $22 \mathrm{~s}$. Worsening balance and gait made shunt intervention a reasonable option. She reported immediately better walking after ventriculoperitoneal shunting (VPS) was placed in early December. The patient left the state later that month but returned to our movement disorder clinic for another examination in October 2019, with MMSE 29/30 and normal gait (average TUG 9.75 s). Her 1-year follow brain MRI shows less contrast enhancement, sulcal hyperintensity, and decreased Evans' ratio from 0.37 to 0.34 (see Fig. 1e-h). The last follow-up evaluation in July 2020 remains the same.

Several case reports of rheumatoid meningitis have emphasized the use of CSF RF in diagnosis. However, the diagnosis of rheumatoid meningitis remains largely dependent on surgical pathology. CSF RF was measured in our patient and was negative, prompting frontal dural and brain biopsy (see Fig. 2). The dural biopsy was essentially unremarkable. Brain biopsy revealed leptomeningitis with chronic inflammation and necrotizing granulomatous formation. The granulomas consisted of central necrosis, rare acute inflammatory cells, and hyaline degeneration surrounded by a chronic inflammatory infiltrate composed of small lymphocytes, plasma cells, histiocytes, and rare giant cells. Underlying brain tissue demonstrated chronic perivascular inflammation and reactive gliosis without vasculitis. Immuno-
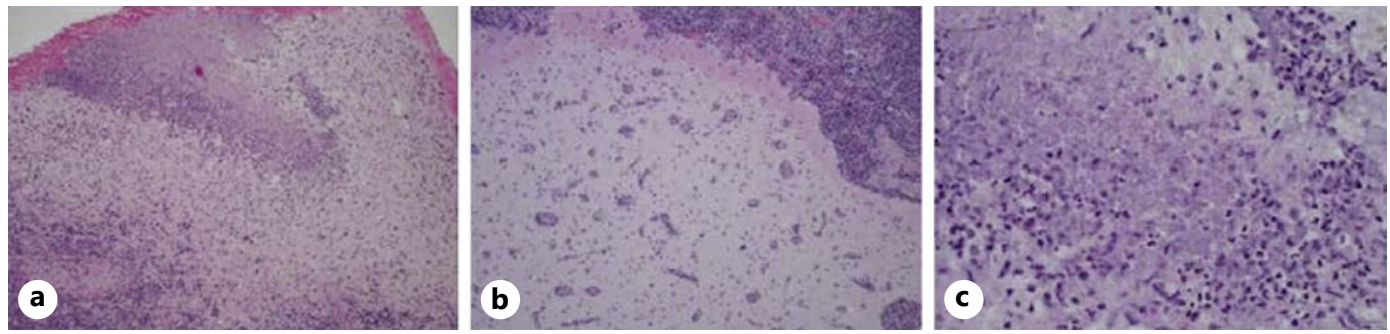

Fig. 2. Meningeal granulomatous inflammation. Sections of leptomeninges with chronic inflammation with necrotizing granulomas formation. The granulomas consist of central necrosis, rare acute inflammatory cells, and hyaline degeneration surrounded by chronic inflammatory infiltrate composed of small lymphocytes, plasma cells, histiocytes, and rare giant cells. Underlying brain shows perivascular chronic inflammation and reactive gliosis without vasculitis. a Necrotizing granulomas. b Meningeal inflammation. c Granuloma.

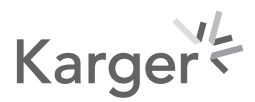


staining demonstrated up to 35-40\% IgG positive plasma cells in highest labeling areas. Gram stain, silver stain, and fluorochrome staining for acid-fast bacilli ruled out underlying infection. Surgical pathology was deemed consistent with rheumatoid meningitis and correlated well with her imaging findings of leptomeningeal disease without significant dural enhancement or brain involvement.

\section{Discussion/Conclusion}

The pathophysiology of NPH is incompletely understood but thought to be related to pressure within the ventricles compressing the brain against the calvarium, reducing cerebral blood flow, particularly in the frontal lobes and subcortical areas [1]. Secondary NPH encompasses all cases of NPH with an identified cause, including meningitis, subarachnoid hemorrhage (SAH), intracerebral hemorrhage, brain tumor, or head trauma. SAH and trauma are the most common and best-studied causes. Meningoencephalitis accounted for $5 \%$ of cases in a review of 1,208 cases. Secondary NPH appears to include an initial acute injury phase of elevated pressure, which causes ventricular enlargement followed by stabilization and pressure normalization. There are two broad categories of pathological processes leading to secondary NPH: increased CSF viscosity and leptomeningeal scarring. Increased viscosity is seen acutely in SAH, meningitis, and tumors. Leptomeningeal fibrosis, arachnoid adhesions, and scarring of the basal cisterns are a late effect of SAH and meningitis. This permanently impairs CSF flow and reabsorption [2].

Rheumatoid meningitis can cause either pachymeningitis or leptomeningitis. Serum RF and CCP are usually elevated but can be normal. In a review of 49 cases since 2010, RF was negative in 5 out of 39 cases with a numerical range of 8-7,900 IU/mL, and CCP was negative in 1 out of 33 with a range of 7-1,448 IU/mL [3]. CSF RF and CCP are thought to be specific but not sensitive - these have only been measured in 5 patients, including ours [3]. The histopathology is characterized most commonly by rheumatoid nodules (68\%), nonspecific inflammatory infiltrates (63\%), and vasculitis (20-37\%) [4]. Granulomatous inflammation is common. High levels of plasma cells in the inflammatory infiltrate can distinguish rheumatoid meningitis from other causes of meningitis. Nodules are the most specific finding and are identical to subcutaneous rheumatoid nodules; however, these are less likely to induce neurologic symptoms directly unless they are large enough to cause mass effect. Leptomeningitis is the most likely pathology to present with focal neurological features and is more commonly implicated in gait abnormality, likely due to fibrosis and adhesions as discussed above [4-6].

The treatment for rheumatoid meningitis is similar to any systemic autoimmunity with CNS involvement, with high-dosed steroids followed by aggressive immune suppression with cyclophosphamide or rituximab. Mortality is historically high. Of note, tissue necrosis factor agents might make CNS involvement worse in rheumatoid meningitis, and methotrexate may cause meningeal rheumatoid nodulosis [7], not present in our case.

Previous reports of clinical NPH or Parkinsonism in rheumatoid meningitis were largely remitted with steroid treatment $[3,8-10]$ (see Table 1 ), with successful escalation to rituximab or cyclophosphamide in two patients. Ours is the second case of rheumatoid meningitis to undergo shunting and the first biopsy-confirmed case. It has been previously hypothesized that vasculitis affecting the basal ganglia is the likely cause of Parkinsonism in rheumatoid meningitis [6], but this remains speculative with the scarcity of cases to date. NPH causing an atypical Parkinsonism may be a more likely etiology in the absence of basal ganglia infarct on MRI.

We decided not to do large volume spinal drainage due to VPS's high response rate in secondary NPH. Outcomes of VPS are much better in secondary NPH with improvement in

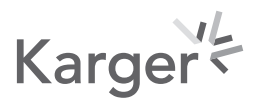


Case Reports in Neurology
Case Rep Neurol 2021;13:434-440

Owens et al.: Secondary NPH in Rheumatoid Meningitis

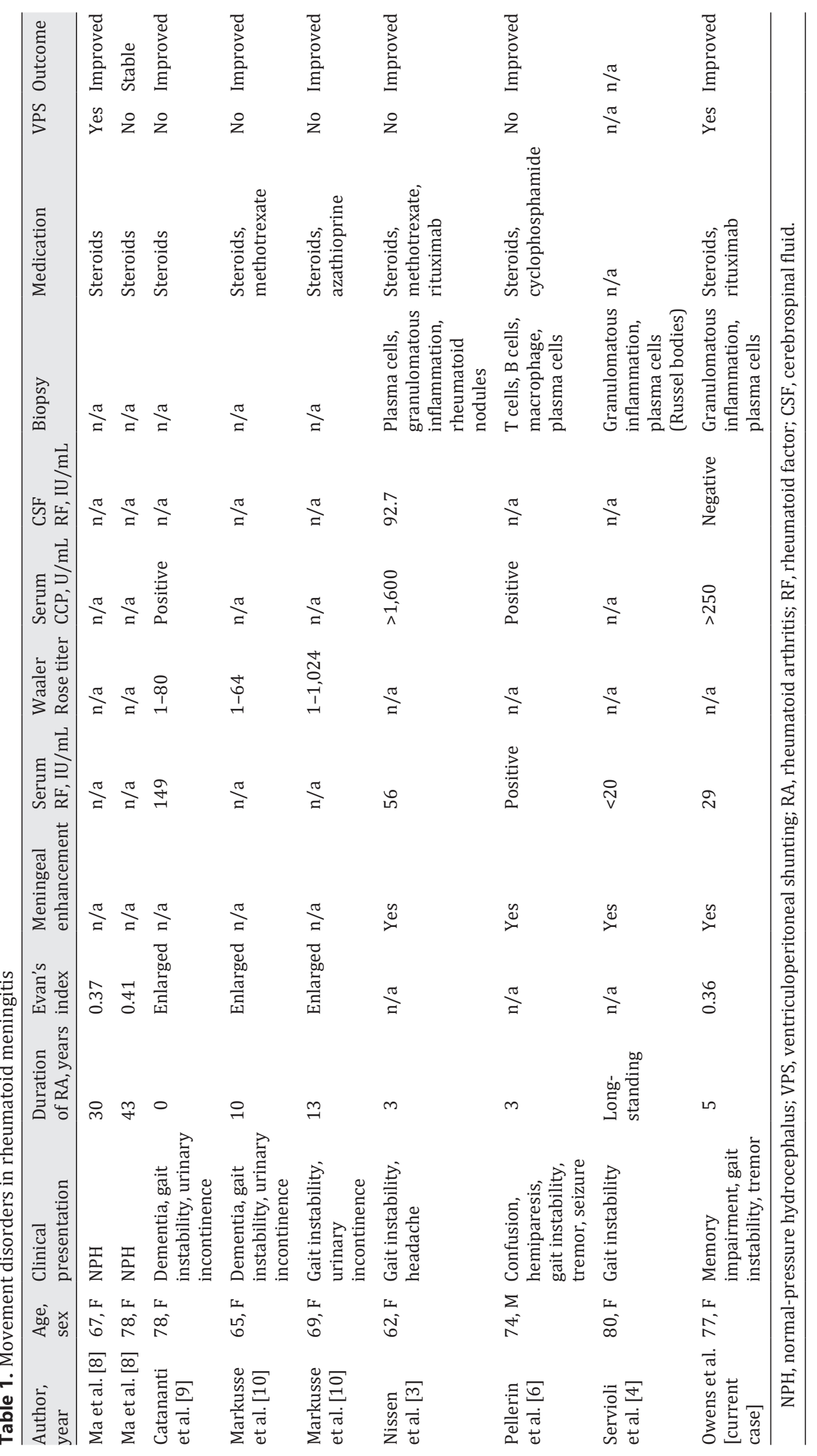


$>70 \%$ of secondary cases (including $>50 \%$ "excellent" recovery) compared to $30-50 \%$ improvement in idiopathic cases reported elsewhere [2]. This trend holds true in secondary NPH associated with autoinflammatory conditions treated with steroids [8]. Our patient's initially reported walk improvement is most likely due to VPS, even though we know that a predictive test showing a good response to large volume tap or an immediate neurological examination after the shunt could have brought more certainty. However, we speculate rituximab has helped maintain a normal gait and mental status beyond one year after the surgery, considering her follow-up brain MRI showing less contrast enhancement and decreased Evans' ratio (see Fig. 1e-h).

\section{Statement of Ethics}

IRB approval was not required for this case report. Written informed consent for write-up and publishing of the case was obtained from the patient including written consent for publication of image files. We confirm that we have read the Journal's position on issues involved in ethical publication and affirm that this work is consistent with those guidelines.

\section{Conflict of Interest Statement}

The authors have no conflicts of interest to declare.

\section{Funding Sources}

No specific funding was received for this work.

\section{Author Contributions}

1. Research project: A. conception, B. organization, C. execution.

2. Statistical analysis: A. design, B. execution, C. review and critique - N/A.

3. Manuscript preparation: A. writing of the first draft, B. review and critique.

4. Literature review.

M. Owens: 1B, 3A, 4; N.T. Gatson: 1A, 1B, 1C, 3B; G. Mongelluzzo: 1A, 1B, 1C, 3B; 0. Goren: 1A, 1B, 1C, 3B; E. Newman: 1A, 1B, 1C, 3B.

\section{References}

1 Oliveira LM, Nitrini R, Roman GC. Normal-pressure hydrocephalus: a critical review. Dement Neuropsychol. 2019;13(2):133-43.

2 Daou B, Klinge P, Tjoumakaris S, Rosenwasser RH, Jabbour P. Revisiting secondary normal pressure hydrocephalus: does it exist? A review. Neurosurg Focus. 2016;41(3):E6.

3 Nissen MS, Nilsson AC, Forsberg J, Milthers J, Wirenfeldt M, Bonde C, et al. Use of cerebrospinal fluid biomarkers in diagnosis and monitoring of rheumatoid meningitis. Front Neurol. 2019;10:666.

4 Servioli MJ, Chugh C, Lee JM, Biller J. Rheumatoid meningitis. Front Neurol. 2011;2:84.

5 Bathon JM, Moreland LW, DiBartolomeo AG. Inflammatory central nervous system involvement in rheumatoid arthritis. Semin Arthritis Rheum. 1989;18(4):258-66.

6 Pellerin D, Wodkowski M, Guiot MC, AlDhukair H, Blotsky A, Karamchandani J, et al. Rheumatoid meningitis presenting with acute Parkinsonism and protracted non-convulsive seizures: an unusual case presentation and review of treatment strategies. Front Neurol. 2019;10:163. 
7 Kampylafka EI, Alexopoulos H, Dalakas MC, Tzioufas AG. Immunotherapies for neurological manifestations in the context of systemic autoimmunity. Neurotherapeutics. 2016;13(1):163-78.

8 Ma B, Wu H, Yin H, Chang J, Wang L, Wang R, et al. Management of hydrocephalus associated with autoimmune diseases: a series of 19 cases. Autoimmunity. 2017;50(7):422-7.

9 Catananti C, Mastropaolo S, Calabrese C, Silveri MC, Onder G. A case of normal-pressure hydrocephalus associated with rheumatoid arthritis. Aging Clin Exp Res. 2010;22(2):189-91.

10 Markusse HM, Hilkens PH, van den Bent MJ, Vecht CJ. Normal pressure hydrocephalus associated with rheumatoid arthritis responding to prednisone. J Rheumatol. 1995;22(2):342-3. 